\title{
Erratum: Tensor Network Simulation of Non-Markovian Dynamics in Organic Polaritons [Phys. Rev. Lett. 121, 227401 (2018)]
}

\author{
Javier del Pino, Florian A. Y. N. Schröder, Alex W. Chin, Johannes Feist, and Francisco J. Garcia-Vidal
}

(Received 27 March 2019; published 18 April 2019)

DOI: 10.1103/PhysRevLett.122.159902

The reorganization energy of the molecular model used in the Letter is actually $\Delta=35.6 \mathrm{meV}$ (a factor of $\pi$ smaller than stated). The necessary corrections are as follows:

(1) The last sentence in the left column on page 2 should read: "For Rhodamine 800 this density is extracted from the spectroscopic measurements in Ref. [34] [...] and reorganization energy $\Delta=\int_{0}^{\infty}\left[J_{v}(\omega) / \pi \omega\right] \mathrm{d} \omega \approx 35.6 \mathrm{meV}$."

(2) In the last paragraph of the right column on page 4, the correct value for $\tau_{\mathrm{RC}}$ is $23 \mathrm{fs}$.

(3) The first paragraph in page 5 should read "In addition to the vibration-free polaritons $| \pm\rangle$, emission bands at intermediate energies are visible in the spectrum. This is interpreted as due to small cavity admixtures to dark states, in line with experimental observations [65,72]." We previously misidentified one of the bands as being at the baremolecule emission frequency, $\omega_{e}-2 \Delta$, which does not apply with the corrected value of $\Delta$.

We have also updated the Supplemental Material to correct the issues related with the error in the value of $\Delta$. None of the conclusions in the main text or supplemental material are affected.

We thank Rui E. F. Silva for bringing this to our attention. 